\title{
Where Does It Break? or: Why the Semantic Web Is Not Just "Research as Usual"
}

\author{
Frank van Harmelen \\ Vrije Universiteit Amsterdam \\ The Netherlands \\ http://www.cs.vu.nl/ frankh/
}

\begin{abstract}
Work on the Semantic Web is all too often phrased as a technological challenge: how to improve the precision of search engines, how to personalise web-sites, how to integrate weakly-structured data-sources, etc. This suggests that we will be able to realise the Semantic Web by merely applying (and at most refining) the results that are already available from many branches of Computer Science. I will argue in this talk that instead of (just) a technological challenge, the Semantic Web forces us to rethink the foundations of many subfields of Computer Science. This is certainly true for my own field (Knowledge Representation), where the challenge of the Semantic Web continues to break many often silently held and shared assumptions underlying decades of research. With some caution, I claim that this is also true for other fields, such as Machine Learning, Natural Language Processing, Databases, and others. For each of these fields, I will try to identify silently held assumptions which are no longer true on the Semantic Web, prompting a radical rethink of many past results from these fields.
\end{abstract}

